УДК $004.02+004.855 .5(6)$

DOI: $10.15827 / 0236-235 X .130 .186-194$

Дата подачи статьи: 20.12.19

\title{
Архитектура системы предсказательного технического обслуживания сложных многообъектных систем в кониепиии индустрии 4.0
}

\author{
Сай Ван Квонг 1, аспирант, svcuonghvktqs@gmail.com
}

M.В. Щербаков ${ }^{1}$, д.m.н., старший научный сотрудник, maxim.shcherbakov@gmail.com

${ }^{1}$ Волгоградский государственный технический университет, 2. Волгоград, 400005, Россия

Правильно сформированная стратегия технического обслуживания и ремонта оборудования играет критическую роль в современных экономических условиях, характеризующихся высоким уровнем конкуренции.

В последнее время в рамках внедрения концепции Индустрия 4.0 в области организации технического обслуживания и ремонта сложных многообъектных систем наиболее перспективными представляются подходы, основанные на использовании передовых методов анализа больших массивов данных на базе инновационных технологий искусственного интеллекта. В основном речь идет о концепции предсказательного технического обслуживания, а именно о создании предсказательных моделей для предотвращения отказов оборудования. Такая стратегия технического обслуживания и ремонта позволяет переходить от грубого планового обеспечения к обеспечению функционирования по состоянию с учетом прогнозирования изменений состояния систем с целью достижения их максимальной производительности при минимальных затратах. В данной работе рассматриваются ключевые элементы для реализации концепции предсказательного технического обслуживания.

В результате авторами предложена архитектура системы предсказательного технического обслуживания сложных многообъектных систем в концепции Индустрии 4.0. Система включает в себя три модуля: оффлайн-анализа накопленных данных, онлайн-анализа потоковых данных и поддержки принятия решений. Основными функциями первых двух модулей являются раннее обнаружение и прогнозирование отказов оборудования на основе обработки сенсорных данных с использованием методов машинного обучения. На основе информации, полученной из модуля онлайн-анализа, в модуле поддержки принятия решений формируются оптимальные решения при выборе стратегии воздействия на оборудование при необходимости. Такие решения соблюдают оптимальный баланс между затратами на проведение технологических воздействий и величиной потенциальных ущербов и рисков от отказа оборудования.

Ключевые слова: интернет вещей, промышленный интернет вещей, предсказательное обслуживание, поддержка принятия решений, искусственный интеллект.

Обеспечение работоспособности оборудования с минимальными затратами всегда является актуальной задачей для различных производств. По статистике, из-за неэффективного обслуживания наблюдается двукратный рост расходов на обеспечение функционирования оборудования [1].

Быстрое развитие промышленности и информационно-коммуникационных технологий обусловило усложнение технических систем и оборудования. Нередко это приводит к нарушению нормального функционирования небольшого объекта инфраструктуры и, таким образом, к нарушению работы всей системы в целом. Отказ машинных агрегатов может повлечь за собой возникновение аварийных ситуаций, сопровождающихся значительным ущербом. Класси- ческие подходы к организации технического обслуживания и ремонта оборудования (ТОиР) (корректирующее и планово-профилактическое техническое обслуживание) в таких условиях в значительной мере теряют свою эффективность и не удовлетворяют требованиям современной промышленности.

В корректирующем техническом обслуживании ТОиР выполняется после возникновения отказа. Данный подход также известен как реактивное техническое обслуживание и подходит только в том случае, если последствия отказа незначительны, время его восстановления небольшое и нет риска безопасности. Однако это невозможно соблюсти одновременно в современных отраслях промышленного производства [2]. 
В планово-профилактическом обслуживании (time-based maintenance - TBM) проведение ТОиР предусматривается по истечении определенного времени, которое определяется календарным интервалом и зависит от условий эксплуатации. Достоинством такого метода по сравнению с реактивным обслуживанием является снижение вероятности внезапного отказа оборудования. Однако эта система не лишена недостатков, поскольку зачастую выполняются ремонты фактически исправного оборудования, то есть проводятся «излишние» ремонты, что приводит к большим затратам [1]. Кроме того, эта система не гарантирует безотказную работу оборудования, поскольку большинство отказов носят случайный характер, о чем свидетельствуют результаты масштабных исследований механизмов отказов, проведенных американской авиакомпанией United Airlines (UAL), военно-морским флотом США и другими организациями [3]. Эти исследования показали, что от 70 до $90 \%$ видов отказов не связаны с наработкой оборудования, то есть отказы не соответствуют представлению, принятому практикой ТВМ.

Анализ работ $[1,4,5]$ позволил сделать вывод, что в современных экономических условиях наиболее эффективной и целесообразной стратегией технического обслуживания является предсказательное обслуживание. В последнее время оно становится все более важным, о чем свидетельствует значительное увеличение количества публикаций по этой теме. На рисунке 1 показана динамика числа публикаций и цитирований по теме «предсказательное обслуживание» в период 2009-2018 гг. Поиск проводился по ключевым словам «предсказательное обслуживание» в БД платформы Web of Science. Рисунок показывает, что в данный период число публикаций и цитирований по теме «предсказательное обслуживание» постоянно увеличивалось.

Основой предсказательного обслуживания является техническое обслуживание по техническому состоянию, то есть оно выполняется только в случае необходимости. Предсказательное обслуживание может быть описано как интеллектуальный способ максимизации доступности машины и относится не только к стратегиям для раннего обнаружения и прогнозирования состояния, ухудшения производительности системы, но и для принятия необходимых воздействий, соответствующих распознаванию и предсказанию этих случаев. Данная стратегия направлена на снижение ко-

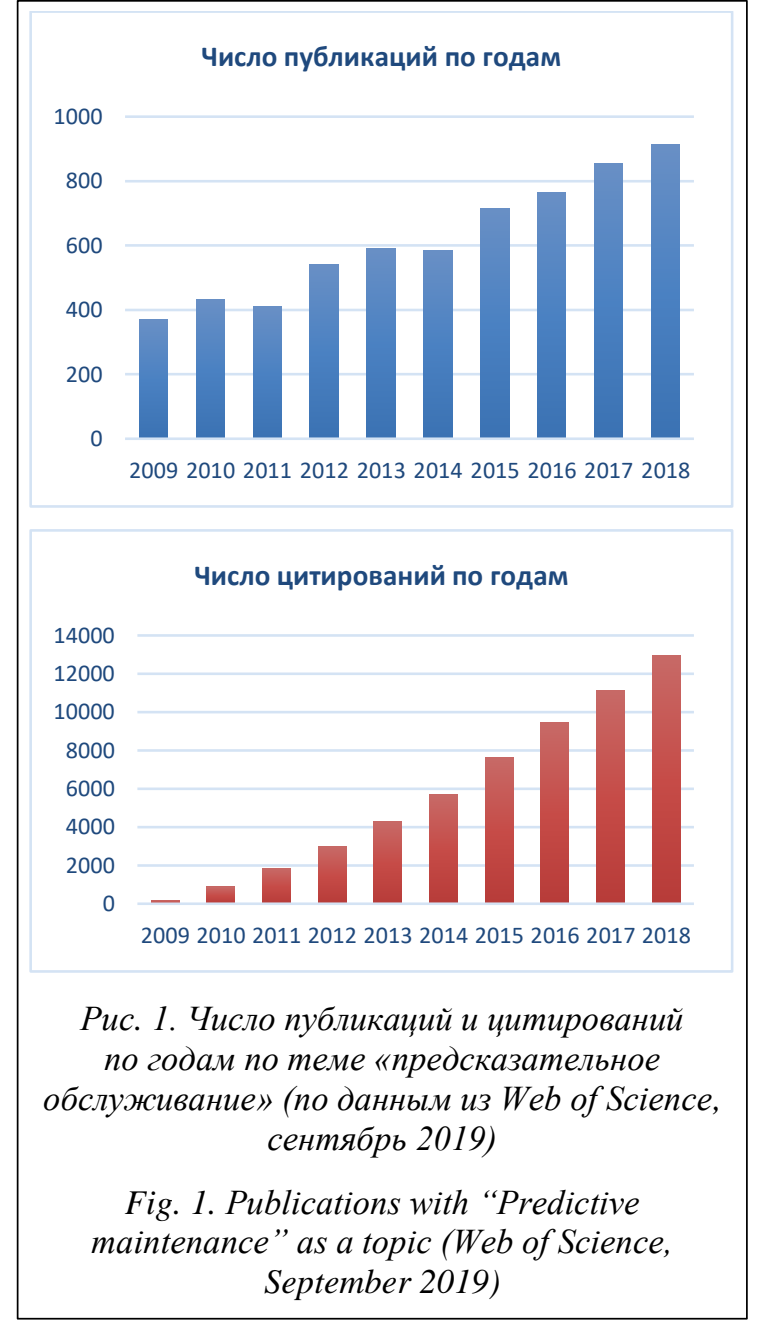

личества необоснованных ремонтных воздействий с целью максимального использования ресурса оборудования за счет разработки и внедрения интеллектуальных систем, выполняющих функции предсказательной аналитики [1]. Такой подход позволяет выполнять обслуживание наиболее эффективным с экономической точки зрения образом и перейти к ремонту по состоянию и к увеличенному межремонтному интервалу. В идеале предсказательное обслуживание позволяет выполнять только правильные работы, сводя к минимуму расход запасных частей, время простоя и время обслуживания.

На рисунке 2 наглядно показана разница между вышеописанными подходами к организации ТОиР (обслуживание по факту отказа, профилактическое и предсказательное обслуживание).

В связи с тенденцией развития умного производства и информационно-коммуникационных технологий компании все чаще стали применять различные типы датчиков и информа- 


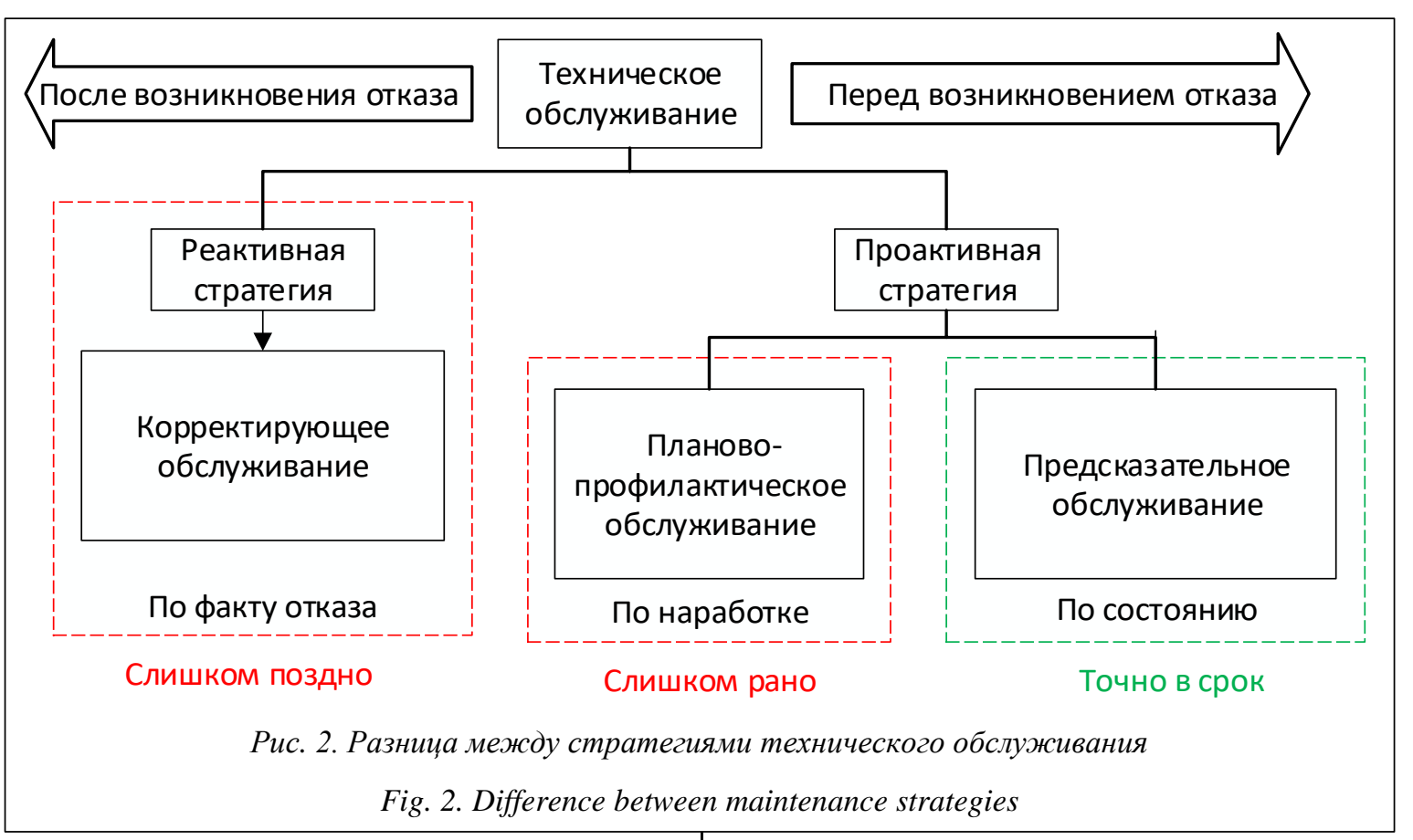

ционных технологий для захвата данных на всех этапах производства. Одновременно такие технологии, как интернет вещей, промышленный интернет вещей, облачные и туманные технологии, искусственный интеллект и интеллектуальный анализ данных, которые присущи Индустрии 4.0, являются решающим фактором для успешной реализации проактивной стратегии технического обслуживания - предсказательного обслуживания.

В данной работе рассматриваются ключевые элементы для реализации проактивной стратегии технического обслуживания - предсказательного обслуживания. В результате предложена архитектура системы предсказательного технического обслуживания на основе обработки накопленных данных и потоковых данных в концепции Индустрии 4.0.

\section{Обзор исследований}

Стандарт EN 13306 определяет предсказательное обслуживание как обслуживание по техническому состоянию, которое осуществляется на основе прогнозов, полученных из анализа и оценки существенных параметров деградации изделия. Его основная цель - диагностика и прогнозирование отказов и неисправностей оборудования.

Для диагностики и прогнозирования отказов в предсказательном обслуживании было представлено много методов. В основном их можно разделить на два типа - методы, осно- ванные на обработке данных (МОД), и методы, основанные на моделях (МОМ) [6].

МОМ используют математические модели для описания физического поведения и процессов деградации оборудования, при этом значения параметров изменяются на основе собранных данных [7, 8]. Наиболее часто используемыми МОМ являются модели марковского процесса [9], винеровского процесса [9], гауссовой смеси [10] и т.д. Основной недостаток МОМ заключается в необходимости регулярной структурной и параметрической оптимизации моделей из-за динамически меняющегося окружения [11]. Кроме того, сложные системы требуют значительных затрат на создание и настройку МОМ, в том числе и с привлечением экспертов [12]. Для преодоления данных недостатков были предложены и в настоящее время активно развиваются методы, основанные на использовании данных [13].

МОД описывают процесс деградации оборудования на основе измеряемых данных с использованием статистических методов или методов искусственного интеллекта [6]. МОД обладают свойствами универсальности, поскольку они абстрагированы от физической природы объектов, не требуют знаний его внутренней структуры и функциональных связей между элементами [14]. Часто используемыми статистическими методами являются регрессионные модели, метод главных компонент, анализ выживаемости и т.д. Пространственные модели состояний, такие как скрытые 
марковские модели или скрытые полумарковские модели, динамические байесовские сети и прочие, также являются широко используемыми статистическими методами. Методы искусственного интеллекта - это обычно различные варианты методов машинного обучения и искусственных нейронных сетей, таких как нейронные сети долгой краткосрочной памяти, сверточные нейронные сети и т.д. Для применения МОМ требуется решить две важные задачи - сбор данных и их обработка.

Сбор данных. В работе [15] описывается сбор данных как процесс получения и хранения полезных данных от целевых физических активов для обслуживания по техническому состоянию. Собранные данные могут быть разделены на два типа - данные мониторинга состояния и событийные данные. В [16] определено, что данные мониторинга состояния, как и любые данные, в том числе эксплуатационные, данные о производительности, об окружающей среде, сигналы деградации, можно использовать для прогнозирования состояния оборудования для предотвращения отказов. Событийные данные включают информацию о том, что произошло с целевыми физическими активами. Событийные данные и данные мониторинга состояния играют исключительную роль в обслуживании по техническому состоянию.

Развитие и удешевление повсеместных систем сбора данных и удешевление систем сбора данных, в том числе в дистанционном режиме и в реальном масштабе времени, позволяют собирать большие объемы данных о многообъектных целевых системах. Однако данные хранятся и обрабатываются в различных и неоднородных системах, таких как компьютеризированные системы управления техническим обслуживанием для ведения учета технического обслуживания, системы мониторинга состояния активов и системы диспетчерского управления и сбора данных SCADA для управления мониторингом процессов и управления активами [17, 18]. В работе [19] утверждается, что объединение компьютеризированных систем управления техническим обслуживанием, систем мониторинга и управления знаниями по техническому обслуживанию является ключевой частью технологии технического обслуживания в будущем.

Обработка данных. В любом приложении, основанном на данных в общем и для предсказательного обслуживания в частности, обработка данных имеет первостепенное значение [20]. Необходимость обработки исходных данных может быть вызвана разными причинами, например, некоторые статистические методы требуют, чтобы все предикторы измерялись в одинаковых единицах. В других случаях качество предсказательных моделей может в значительной мере зависеть от характера распределения данных или наличия выбросов. В общем, процесс обработки данных включает в себя следующие шаги: очистка данных, обработка пропущенных значений, нормализация данных, преобразование данных, инженерия признаков.

Основная цель очистки данных - выявление и удаление ошибок, выбросов и повторяющихся данных для улучшения их качества. Существует огромное количество методов обнаружения выбросов, такие как статистические, машинного обучения, подмены задачи, метрические методы и другие.

Большинство алгоритмов машинного обучения очень чувствительны к шкалированию данных. Поэтому для получения максимальной точности от алгоритмов машинного обучения чаще всего осуществляется нормализация или преобразование исходных данных перед запуском алгоритмов. Некоторыми известными методами преобразования данных являются преобразование по методу Вох-Сох, логарифмическое преобразование, нормализация min$\max$, центрирование и т.д. [21].

Часто в реальных данных присутствуют пропуски, в результате чего возникает необходимость обработки этих пропусков. Существуют два подхода к решению данной задачи: игнорироваение объектов, имеющих пропуски, и замена специальными значениями с помощью различных методов, таких как метод ближайших соседей, метод случайного леса, метод k средних и другие [22].

Инженерия признаков является самым важным и творческим процессом отбора и создания признаков с целью поиска оптимальных подмножеств, на которых построенная модель дает наилучшее качество. Для этой цели используются методы рекурсивного исключения признаков, главных компонент и другие [23].

\section{Предлагаемая архитектура системы предсказательного технического} обслуживания в концепции Индустрии 4.0

На рисунке 3 отображена структура предложенной системы предсказательного технического обслуживания на основе обработки накопленных данных и потоковых данных в 


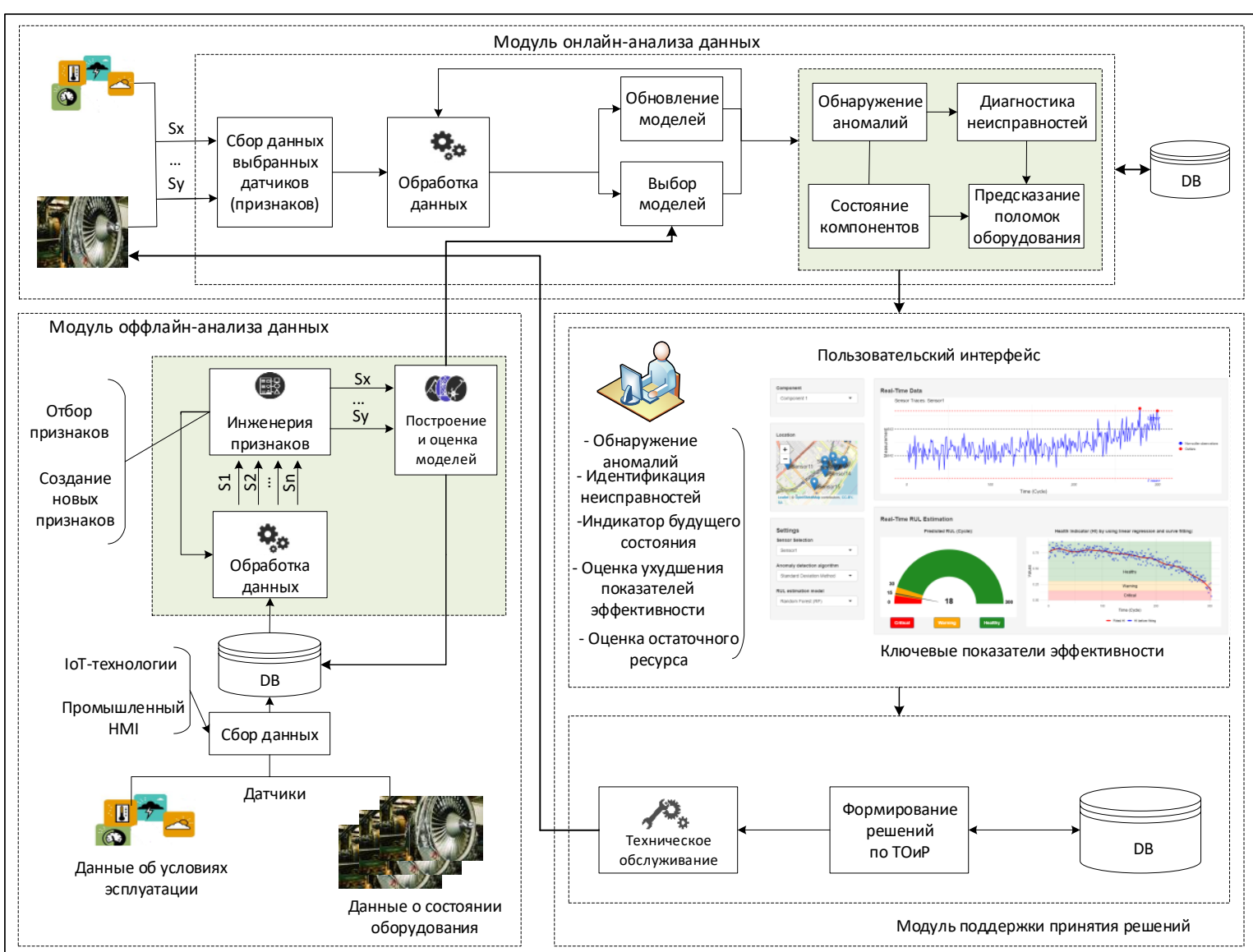

Рис. 3. Предложенная архитектура системы предсказательного технического обслуживания

Fig. 3. Proposed architecture of predictive maintenance system

концепции Индустрии 4.0, которая состоит из трех методологических модулей: оффлайнанализа данных, онлайн-анализа данных, поддержки принятия решений.

Первым важным элементом для развертывания этой системы является процесс мониторинга и сбора данных о поведении анализируемой системы. Этот процесс осуществляется на основе технологий интернета вещей или промышленного интернета вещей. Для обеспечения работы предложенной системы могут использоваться данные мониторинга состояния оборудования и эксплуатационных условий, такие как вибрация от акселерометров, температура и т.д., а также событийные данные о неисправностях, отказах, воздействиях по техническому обслуживанию и т.д. Перед сбором данных важно определить: а) критические компоненты системы (эта задача выполняется на основе различных видов анализа, таких как функциональный анализ, дисфункциональный анализ, анализ критичности, накопленный опыт и другие [24]); б) физические параметры, необходимые для наблюдения; в) датчики для отслеживания процесса деградации оборудования.

После сбора полученные данные будут храниться в системах БД для последующей обработки.

Модуль оффлайн-анализа данных осуществляет анализ исторически накопленных данных, полученных из однотипных систем с использованием различных алгоритмов машинного обучения, глубоких нейронных сетей и технологий инженерии признаков, преобразования данных для формирования моделей, позволяющих раннее обнаружение и диагностику неисправностей, прогнозирование остаточного ресурса оборудования и т.д. Эти модели оцениваются и выбираются для последующего использования в модуле онлайн-анализа данных.

Известно много подходов искусственного интеллекта, используемых для создания предсказательных моделей. В работе [1] были исследованы различные алгоритмы для прогнозирования остаточного ресурса, такие как метод опорных векторов (SVM), деревья решений 
(CART), случайный лес (RF), экстремальный метод градиентного бустинга (XGBoost), сети долгой краткосрочной памяти (Long short-term memory, LSTM), сверточные нейронные сети (Convolutional Neural Network, CNN) и т.д. В работе [25] изучены алгоритмы для раннего обнаружения неисправностей для предсказательного обслуживания, такие как скрытые марковские модели, модель ARIMA, LSTMавтоэнкодеры и другие.

Модуль онлайн-анализа данных осуществяет сбор новых данных в реальном времени только из критических компонентов, выбранных на основе анализа в модуле оффлайн-анализа данных. Выбранные из модуля оффлайнанализа данных модели используются для определения и прогнозирования состояния оборудования в реальном времени.

Однако эти модели в процессе использования на потоковых данных в реальном времени могут устареть в силу многих факторов, например, возможности возникновения новых типов неисправностей, на которых модели не обучались в прошлом. Поэтому возникает необходимость переобучения и обновления моделей. Модуль онлайн-анализа данных предложенной системы предусматривает решение и этой проблемы.

Модуль принятия решений по ТОИР. Оcновной целью этого модуля является оптимальное планирование воздействий по ТОиР. Информация, полученная в процессе дигностики и прогностики в модуле онлайн-анализа данных, используется для выработки рекомендаций по оптимальному использованию оборудования в режиме онлайн. В этом модуле предоставляется пользовательский интерфейс, обеспечивающий передачу информации между пользователем-человеком и программно-аппаратными компонентами системы о результатах процесса анализа (обнаружение/диагностика/ прогностика), а также визуализации потоковых данных в реальном времени. На их основе при необходимости формируются оптимальные воздействия по ТОиР.

\section{Заключение}

В данной работе рассматриваются ограничения классических подходов (корректирующее и профилактическое обслуживание) к организации ТОиР и преимущества проактивной стратегии технического обслуживания на основе обработки накопленных и потоковых данных - предсказательного обслуживания. Анализируются ключевые элементы для реализации предсказательного обслуживания. В результате предложена архитектура интеллектуальной системы технического обслуживания сложных многообъектных систем в концепции Индустрии 4.0. Эта системная архитектура учитывает предварительный оффлайн-анализ собранных накопленных и онлайн-анализ потоковых данных для раннего обнаружения возникновения возможных отказов, прогнозирования остаточного ресурса оборудования.

Дальнейшая работа будет направлена на последующее развитие нескольких модулей архитектуры системы и их развертывание в реальных промышленных производственных подразделениях для технического обслуживания сложных многообъектных систем.

Работа выполнена при поддержке РФФИ, проект № 19-47-340010 r_a.

\section{Лumepamypa}

1. Сай Ван Квонг, Щербаков М.В. Метод прогнозирования остаточного ресурса на основе обработки данных многообъектных сложных систем // Прикаспийский журнал: управление и высокие технологии. 2019. № 1. С. 33-44.

2. Wang Y., Deng C., Wu J., Wang Y., Xiong Y. A corrective maintenance scheme for engineering equipment. Engineering Failure Analysis, 2014, no. 36, pp. 269-283.

3. Allen T.M. Us navy analysis of submarine maintenance data and the development of age and reliability profiles. URL: https:/www.plant-maintenance.com/articles/SubmarineMaintenanceDataRCM.pdf (дата обращения: 15.12.2019).

4. Khoury E., Deloux E., Grall A., Berenguer C. On the use of time-limited information for maintenance decision support: a predictive approach under maintenance constraints. Mathematical Problems in Engineering, 2013, no. 2, pp. 1-11.

5. Huang Z.Y., Xu Z.G., Wang W.H., and Sun Y.X. Remaining useful life prediction for a nonlinear heterogeneous Wiener process model with an adaptive drift. IEEE Trans. Rel., 2015, vol. 64, no. 2, pp. 687-700.

6. Ying Peng, Ming Dong, Ming Jian Zuo. Current status of machine prognostics in condition-based maintenance: a review. Int. J. Adv. Manuf. Technol., 2010, no. 50, pp. 297-313. DOI: 10.1007/s00170-0092482-0. 
7. Hanachi H., Liu J., Banerjee A., Chen Y., Koul A. A physics-based modeling approach for performance monitoring in gas turbine engines. IEEE Transactions on Reliability, 2015, vol. 64, no. 1, pp. 197-205.

8. Dui H., Si S., Zuo M., Sun S. Semi-Markov process-based integrated importance measure for multistate systems. IEEE Transactions on Reliability, 2015, vol. 2, no. 64, pp. 754-765.

9. Khoury E., Deloux E., Grall A., Berenguer C. On the use of time-limited information for maintenance decision support: a predictive approach under maintenance constraints. Mathematical Problems in Engineering, 2013, no. 2, pp. 1-11. DOI: 10.1155/2013/983595.

10. Zhang L., Li X., Yu J. A review of fault prognostics in condition based maintenance. Proc. of SPIE, 2006, vol. 6357. DOI: 10.1117/12.717514.

11. Liu J., Saxena A., Goebel K., Saha B., Wang W. An adaptive recurrent neural network for remaining useful life prediction of lithiumion batteries. Proc. Annual Conf. of the Prognostics and Health Management Society, 2010, pp. 1-9.

12. Yaguo L., Naipeng Li, Gontarz S., Lin J., Radkowski S., Dybala J. A model-based method for remaining useful life prediction of machinery. IEEE Transactions on Reliability, 2016, no. 65, pp. 1-13.

13. Goebel K., Saha B., Saxena A., Celaya J.R., Christophersen J.P. Prognostics in battery health management. IEEE Instrumentation \& Measurement Magazine, 2008, vol. 8, pp. 33-40.

14. Liu J., Wang W., Golnaraghi F. A multi-step predictor with a variable input pattern for system state forecasting. Mech. Syst. Signal Process., 2009, vol. 23, pp. 1586-1599.

15. Jardine A.K.S., Lin D., Banjevic D. A review on machinery diagnostics and prognostics implementing condition-based maintenance. Mech. Syst. Signal Process., 2006, vol. 7, no. 20, pp. 1483-1510. DOI: 10.1016/j.ymssp.2005.09.012.

16. Si X.S., Wang W., Hu C.H., Zhou D.H. Remaining useful life estimation - A review on the statistical data driven approaches. Europ. J. of Operational Research, 2011, no. 213, pp. 1-14. DOI: 10.1016/j.ejor. 2010.11.018.

17. Galar D., Kumar U., Juuso E., Lahdelma S. Fusion of maintenance and control data: a need for the process. Proc. 18th World Conf. on Nondestructive Testing, 2012. URL: http://www.diva-portal.org/smash/get/diva2:1001796/FULLTEXT01.pdf (дата обращения: 15.12.2019).

18. Galar D., Kans M., Schmidt B. Big data in asset management: knowledge discovery in asset data by the means of data mining. Proc. 10th WCEAM, Cham, Springer, 2015, pp. 161-171. DOI: 10.1007/978-3-31927064-7.

19. Bjorling S.E., Baglee D., Galar D., Singh S. Maintenance knowledge management with fusion of CMMS and CM. Proc. DMIN Intern. Conf. Data Mining, Las Vegas, 2013. URL: https://www. researchgate.net/publication/311844431_Maintenance_Knowledge_Management_with_Fusion_of_CMMS_ and_CM (дата обращения: 15.12.2019).

20. Zhang L., Xiong G., Liu H., Zou H., Guo W. Bearing fault diagnosis using multi-scale entropy and adaptive neuro-fuzzy inference. Expert Syst. Appl., 2010, no. 37, pp. 6077-6085.

21. Cernuda C. On the relevance of preprocessing in predictive maintenance for dynamic systems. In: Lughofer E., Sayed-Mouchaweh M. (Eds.). Predictive Maintenance in Dynamic Systems. Cham, Springer, 2019, pp. 53-93. DOI: 10.1007/978-3-030-05645-2_3.

22. Handling missing values in data. URL: https://r-analytics.blogspot.com/2017/01/blog-post. html\#.XO6_nIgzZPY (дата обращения: 15.12.2019).

23. Feature selection with the Caret R package. URL: https://machinelearningmastery.com/feature-selection-with-the-caret-r-package/ (дата обращения: 15.12.2019).

24. Gouriveau R., Medjaher K., Zerhouni N. From prognostics and health systems management to predictive maintenance 1: Monitoring and prognostics. Wiley, 2016, 182 p. DOI: 10.1002/9781119371052.

25. Kuzin T., Borovicka T. Early Failure Detection for Predictive Maintenance of Sensor Parts. CEUR Workshop Proc., 2016, pp. 123-130.

\section{Architecture of predictive maintenance system of complex multi-object systems in Industry 4.0 concept}

Sai Van Cuong ${ }^{1}$, Postgraduate Student, svcuonghvktqs@gmail.com M.V.Shcherbakov ${ }^{1}$, Dr.Sc. (Engineering), Senior Researcher, maxim.shcherbakov@gmail.com

${ }^{1}$ Volgograd State Technical University, Volgograd, 400005, Russian Federation 
Abstract. Properly formed strategy of maintenance of equipment plays a critical role in modern economic conditions characterized by crisis phenomena and high levels of competition.

Recently, as part of the implementation of the concept of Industry 4.0 in the field of maintenance of complex multi-object systems, the most promising approaches are based on the use of advanced methods for analyzing large data based on innovative artificial intelligence technologies. It is mainly about the concept of predictive maintenance (PdM), namely the creation of predictive models to prevent equipment failures. This maintenance strategy allows to move from time-based maintenance to condition based maintenance, taking into account the prediction of changes in system states in order to achieve their maximum performance at minimal cost. Therefore, this paper discusses the key elements for implementing the PdM strategy.

As a result, an architecture for predictive maintenance of complex multi-object systems in the concept of Industry 4.0 is proposed. The proposed system includes three modules: an offline-analysis module for accumulated data, an online-analysis module for streaming data, and a decision support module. The main functions of the first two modules are early detection and prediction of equipment failure based on machine learning methods. Based on the information received from the online analysis module, the decision support module generates optimal decisions when choosing a strategy for influencing the equipment, if necessary. Such solutions maintain an optimal balance between the cost of performing technological impacts and the magnitude of potential damages and risks from equipment failure.

Keywords: Internet of thing, industrial Internet of things, predictive maintenance, decision making support, artificial intelligence.

Acknowledgements. This work was with financial support by RFBR, project no. 19-47-340010 r_a.

\section{References}

1. Sai Van Cuong, Shcherbakov M.V. A data-driven method for remaining useful life prediction of multiple-component systems. Caspian J.: Control and High Technologies, 2019, no. 1, pp. 33-44.

2. Wang Y., Deng C., Wu J., Wang Y., Xiong Y. A corrective maintenance scheme for engineering equipment. Engineering Failure Analysis, 2014, no. 36, pp. 269-283.

3. Allen T.M. Us Navy Analysis of Submarine Maintenance Data and the Development of Age and Reliability Profiles. Available at: https://www.plant-maintenance.com/articles/SubmarineMaintenanceDataRCM.pdf (accessed December 15, 2019).

4. Khoury E., Deloux E., Grall A., Berenguer C. On the use of time-limited information for maintenance decision support: a predictive approach under maintenance constraints. Mathematical Problems in Engineering, 2013, no. 2, pp. 1-11.

5. Huang Z.Y., Xu Z.G., Wang W.H., and Sun Y.X. Remaining useful life prediction for a nonlinear heterogeneous Wiener process model with an adaptive drift. IEEE Trans. Rel., 2015, vol. 64, no. 2, pp. 687-700.

6. Ying Peng, Ming Dong, Ming Jian Zuo. Current status of machine prognostics in condition-based maintenance: a review. Int. J. Adv. Manuf. Technol., 2010, no. 50, pp. 297-313. DOI: 10.1007/s00170-0092482-0.

7. Hanachi H., Liu J., Banerjee A., Chen Y., Koul A. A physics-based modeling approach for performance monitoring in gas turbine engines. IEEE Transactions on Reliability, 2015, vol. 64, no. 1, pp. 197-205.

8. Dui H., Si S., Zuo M., Sun S. Semi-Markov process-based integrated importance measure for multistate systems. IEEE Transactions on Reliability, 2015, vol. 2, no. 64, pp. 754-765.

9. Khoury E., Deloux E., Grall A., Berenguer C. On the use of time-limited information for maintenance decision support: a predictive approach under maintenance constraints. Mathematical Problems in Engineering, 2013, no. 2, pp. 1-11. DOI: 10.1155/2013/983595.

10. Zhang L., Li X., Yu J. A review of fault prognostics in condition based maintenance. Proc. of SPIE, 2006, vol. 6357. DOI: 10.1117/12.717514.

11. Liu J., Saxena A., Goebel K., Saha B., Wang W. An adaptive recurrent neural network for remaining useful life prediction of lithiumion batteries. Proc. Annual Conf. of the Prognostics and Health Management Society, 2010, pp. 1-9.

12. Yaguo L., Naipeng Li, Gontarz S., Lin J., Radkowski S., Dybala J. A model-based method for remaining useful life prediction of machinery. IEEE Transactions on Reliability, 2016, no. 65, pp. 1-13.

13. Goebel K., Saha B., Saxena A., Celaya J.R., Christophersen J.P. Prognostics in battery health management. IEEE Instrumentation \& Measurement Magazine, 2008, vol. 8, pp. 33-40.

14. Liu J., Wang W., Golnaraghi F. A multi-step predictor with a variable input pattern for system state forecasting. Mech. Syst. Signal Process., 2009, vol. 23, pp. 1586-1599. 
15. Jardine A.K.S., Lin D., Banjevic D. A review on machinery diagnostics and prognostics implementing condition-based maintenance. Mech. Syst. Signal Process., 2006, vol. 7, no. 20, pp. 1483-1510. DOI: 10.1016/j.ymssp.2005.09.012.

16. Si X.S., Wang W., Hu C.H., Zhou D.H. Remaining useful life estimation - A review on the statistical data driven approaches. Europ. J. of Operational Research, 2011, no. 213, pp. 1-14. DOI: 10.1016/j.ejor.2010. 11.018.

17. Galar D., Kumar U., Juuso E., Lahdelma S. Fusion of maintenance and control data: a need for the process. Proc. 18th World Conf. on Nondestructive Testing, 2012. Available at: http://www.diva-portal.org/ smash/get/diva2:1001796/FULLTEXT01.pdf (accessed December 15, 2019).

18. Galar D., Kans M., Schmidt B. Big data in asset management: knowledge discovery in asset data by the means of data mining. Proc. 10th WCEAM, Cham, Springer, 2015, pp. 161-171. DOI: 10.1007/978-3-31927064-7.

19. Bjorling S.E., Baglee D., Galar D., Singh S. Maintenance knowledge management with fusion of CMMS and CM. Proc. DMIN Intern. Conf. Data Mining, Las Vegas, 2013. Available at: https://www. researchgate.net/publication/311844431_Maintenance_Knowledge_Management_with_Fusion_of_CMMS_ and_CM (accessed December 15, 2019).

20. Zhang L., Xiong G., Liu H., Zou H., Guo W. Bearing fault diagnosis using multi-scale entropy and adaptive neuro-fuzzy inference. Expert Syst. Appl., 2010, no. 37, pp. 6077-6085.

21. Cernuda C. On the relevance of preprocessing in predictive maintenance for dynamic systems. In: Lughofer E., Sayed-Mouchaweh M. (Eds.). Predictive Maintenance in Dynamic Systems. Cham, Springer, 2019, pp. 53-93. DOI: 10.1007/978-3-030-05645-2_3.

22. Handling Missing Values in Data. Available at: https://r-analytics.blogspot.com/2017/01/blog-post. html\#.XO6_nIgzZPY (accessed December 15, 2019).

23. Feature Selection with the Caret $R$ Package. Available at: https://machinelearningmastery.com/featureselection-with-the-caret-r-package/ (accessed December 15, 2019).

24. Gouriveau R., Medjaher K., Zerhouni N. From Prognostics and Health Systems Management to Predictive Maintenance 1: Monitoring and Prognostics. Wiley, 2016, 182 p. DOI: 10.1002/9781119371052.

25. Kuzin T., Borovicka T. Early Failure Detection for Predictive Maintenance of Sensor Parts. CEUR Workshop Proc., 2016, pp. 123-130.

\section{Для цитирования}

Сай Ван Квонг, Щербаков М.В. Архитектура системы предсказательного технического обслуживания сложных многообъектных систем в концепции Индустрии 4.0 // Программные продукты и системы. 2020. Т. 33. № 2. С. 186-194. DOI: 10.15827/0236-235X.130.186-194.

\section{For citation}

Sai Van Cuong, Shcherbakov M.V. Architecture of predictive maintenance system of complex multiobject systems in Industry 4.0 concept. Software \& Systems, 2020, vol. 33, no. 2, pp. 186-194 (in Russ.). DOI: 10.15827/0236-235X.130.186-194. 